Escuela de Ciencias de la Educación
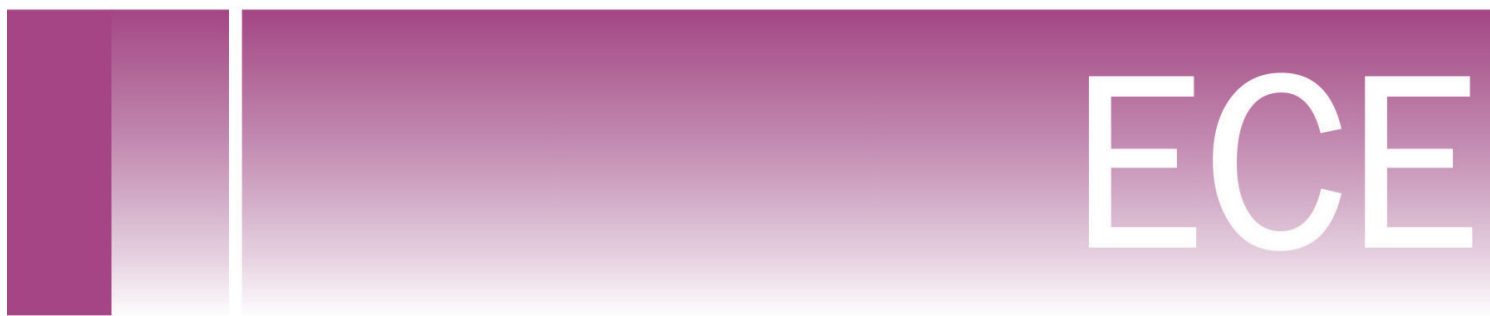



\title{
VISIBILIDAD DE LOS ACTORES EN UNA COMUNIDAD DE APRENDIZAJE, UNA MIRADA DESDE EL ANÁLISIS DE REDES SOCIALES
}

\author{
Juan Carlos Hernández Barrero ${ }^{1}$ y \\ Widman Said Valbuena Buitrago ${ }^{2}$
}

\begin{abstract}
RESUMEN
En el ámbito escolar la configuración de redes sociales es un factor claramente identificable. Hasta el momento existen estudios incipientes que relacionan estas configuraciones sociales con los procesos de aprendizaje, y por ende, escasas estrategias dentro de la dinámica escolar que aporten a la generación de lazos de colaboración.
\end{abstract}

La visibilidad y los dispositivos que la hacen posible han estado presentes en el contexto escolar durante mucho tiempo, pero paradójicamente no se han realizado investigaciones serias desde una perspectiva pedagógica de este fenómeno en relación con el desarrollo conceptual, el aprendizaje y trabajo colaborativo.

Se presenta aquí un acercamiento al estudio pedagógico de la visibilidad, apoyado en el uso de TIC, que muestra la incidencia de ésta en el desarrollo conceptual y el trabajo colaborativo con el perfeccionamiento de una metodología mixta basada en un diseño cuasiexperimental, el análisis de redes sociales, ARS, y el análisis cualitativo y cuantitativo del discurso.

Se ha encontrado, además, que el uso pedagógico de la visibilidad con apoyo en TIC para publicar el trabajo académico y propiciar la interacción de los actores escolares tiene importantes efectos sobre la configuración de redes académicas de los estudiantes y, por tanto, configura escenarios para la constante negociación de significados, lo cual tiene efecto directo en el desarrollo conceptual de los participantes, a diferencia de lo que se observa en un grupo no intervenido con una estrategia pedagógica basada en la visibilidad y apoyada en TIC.

Palabras clave: visibilidad y TIC, comunidad de aprendizaje, redes sociales, desarrollo conceptual, trabajo colaborativo.

1 Coordinador de investigación ZCBC, unAD, Magister en Tecnologías de la Información Aplicadas a la Educación, miembro del grupo de investigación Gestión Vital. Código de registro en Colciencias: COL0056645, Línea de investigación: Visibilidad y gestión de conocimiento en la educación, Red de Investigación: Visibilidad y gestión del Conocimiento.

2 Profesor de la Universidad Nacional de Colombia, Licenciado en Diseño Tecnológico, Especialista en pedagogía del diseño, Magister en Hábitat, miembro del grupo de investigación Gestión Vital. 


\begin{abstract}
At school level, the configuration of social networks is a clearly identifiable factor. So far there exist incipient studies that relate these social configurations to the learning processes, and therefore, there are few strategies within the school dynamics contributing to the creation of partnerships.

The visibility and devices that make it possible have been present in the school for a long time, but paradoxically there have been no serious studies from a pedagogical perspective of this phenomenon in relation to the conceptual development, learning and collaborative work.

This paper presents a pedagogical approach to the study of visibility supported on the use of ICT that shows the impact on the conceptual development and collaborative work with the development of a mixed methodology based on a cuasiexperimental design, social network analysis SNA and qualitative and quantitative analysis of discourse.

Pedagogical use of visibility supported on ICT to publish scholarly work and encouraging the interaction of the school actors has important effects on shaping students' academic networks and this creates scenarios for constant negotiation of meanings, which has direct effect in the conceptual development of the participants, unlike what is observed in a group not involved with a teaching strategy based on the visibility and supported by ICT
\end{abstract}

Key words: visibility and ICT, Information and Communication Technologies, community learning, social network, conceptual development, and collaborative work.

\title{
INTRODUCCIÓN
}

En el ámbito escolar, la configuración de redes sociales es un factor claramente identificable. Hasta el momento existen estudios incipientes que relacionan estas configuraciones sociales con los procesos de aprendizaje, y por ende, escasas estrategias dentro de la dinámica escolar que aporten a la generación de lazos de colaboración.

En las aulas de clase, con frecuencia, las producciones de los estudiantes y aún las de los profesores son ignoradas - invisibles - El trabajo del estudiante, en casi todas las ocasiones, es una producción conocida solo por su profesor y por quien la hace, y los trabajos en grupo quedan en el ámbito de los miembros de ese colectivo.

Esta situación tiene consecuencias en la comunidad que pierde condiciones interesantes para tejer una red social con identidad alrededor de las áreas de conocimiento y la capacidad para actuar colaborativamente en la superación de retos comunes.

La incursión de la Internet en la sociedad contemporánea está acompañada de fenómenos relacionados con la visibilidad que van desde la publicidad hasta la formación de comunidades de interés alrededor de infinidad de temas. También en la escuela este fenómeno se evidencia. 
Una aproximación pedagógica al uso de la informática para mejorar la calidad de la educación y que no ha sido investigada aún de manera sistemática es el tratamiento pedagógico de la visibilidad. En el presente estudio se aborda como un fenómeno que, aunque no es exclusivo, sí hace parte de la dinámica humana y sus implicaciones en la interacción entre actores de una comunidad académica y su incidencia en el aprendizaje.

Los principales enfoques asimilados que orientan esta investigación son:

Según Vigotsky (1978), la interacción con el medio de los individuos genera cambios ya sea de aceleración, retardo, transformación del orden del autodesarrollo, etc. En esta lógica, la presente investigación se sustenta desde los cambios que se evidencian en los estudiantes a partir del efecto de hacer visibles sus producciones, encontrando que los estudiantes vinculados con la estrategia de visibilidad cualifican sus producciones debido a su empeño en visibilizarlo y a los aportes de sus compañeros.

Fisherman (2004) propone que la visibilidad ha sido estudiada como un elemento asociado al reconocimiento que se hace de un individuo en una comunidad. El reconocimiento social se relaciona, en algunos casos, con el desarrollo individual. Por ejemplo, en un estudio sobre identidad espiritual, e identidad personal, las adolescentes aparecen influenciadas por el reconocimiento social mas no los jóvenes de la misma edad.

Para Bandura (1986), la visibilidad y el prestigio social que conlleva el trabajo colaborativo que se deriva de las interacciones comunicativas alcanzan iniciativas exitosas.

Maldonado et al., (2008) estudian el fenómeno de la visibilidad y lo relacionan con el desarrollo científico. La abordan desde las producciones derivadas de procesos investigativos y su impacto en la comunidad científica y encuentran que las producciones que no son visibles, no son reconocidas y no aportan al desarrollo de una institución. Por otro lado, también hallan que el fenómeno de la visibilidad activa la dinámica de la inclusión social en actividades de trabajo colaborativo y en eventos de evaluación entre pares.

\section{Propósitos de la investigación}

El objetivo general que se persigue con esta investigación se centra en determinar si la visibilidad de los productos del trabajo académico, en los estudiantes de una comunidad académica escolar, actúa como activador del desarrollo de competencias conceptuales y del trabajo colaborativo.

Este propósito nuclear se apoya en una ruta metodológica que busca llegar inicialmente a reconocer el estado original de las variables (visibilidad, trabajo colaborativo y desarrollo conceptual).

Simultáneamente, se plantea el diseño e implementación de una estrategia de visibilidad que muestre los resultados del trabajo académico del grupo de estudiantes con los cuales se lleva 
a cabo esta investigación y el apoyo a ésta estrategia con el modelamiento de un dispositivo (Software) de visibilidad que contenga los atributos propios de un mediador de la interacción entre el docente y sus estudiantes y entre éstos mismo como pares académicos.

Posteriormente, se plantea el correspondiente análisis del comportamiento de las variables dependientes con la implementación de la estrategia de visibilización, comparando las probables diferencias que expresan el desarrollo conceptual y el trabajo colaborativo entre un grupo experimental y un grupo control.

Finalmente, se realiza el trabajo que permitirá determinar el efecto que tiene la visibilidad en el trabajo colaborativo y en el desarrollo conceptual.

\section{METODOLOGÍA}

La población objeto de este estudio está constituida por los estudiantes de la I.E. Escuela Normal Superior Distrital María Montessori, particularmente aquellos del área de Tecnología e Informática. La muestra son 80 estudiantes de dos cursos de grado noveno, uno de éstos como grupo experimental con 40 estudiantes y el otro como grupo control, con igual número de participantes, lo cual ubica el diseño metodológico como cuasiexperimental: Campbell, (1993).

\section{Definiciones Operacionales}

Como se comentó con anterioridad, las variables que se miden en la presente investigación son: la visibilidad, el desarrollo conceptual, y el trabajo colaborativo. Se determinó que la variable independiente es la Visibilidad y las dependientes son el trabajo colaborativo y el desarrollo conceptual. De esta manera se pretende estudiar la incidencia que tiene la visibilidad en la construcción de conceptos y en el trabajo colaborativo.

Las formas de medición de cada variable se proponen de la siguiente manera:

\section{Definición operacional de Visibilidad}

Se entiende la visibilidad como un fenómeno, que afecta la dinámica social, en el que los individuos adquieren un reconocimiento mutuo a partir de mostrar sus atributos. En el contexto escolar existen espacios de socialización donde operan dispositivos de visibilidad con los que los estudiantes tienen la posibilidad de colectivizar los productos de su trabajo escolar. Para citar algunos ejemplos existen: periódico institucional, carteleras murales, exposiciones orales, ferias de exposición, sitios Web, participación en clase y otros.

Al tener en cuenta esta característica del contexto escolar se considera posible medir la visibilidad de sus productos utilizando la frecuencia con la que miden los espacios de socialización, la cantidad de dispositivos operantes dentro de esos espacios y la descripción de cómo son usados éstos por los individuos. 


\section{Definición operacional del trabajo colaborativo}

Se entiende el trabajo colaborativo en la escuela como una actividad de negociación en la cual sus participantes, a partir del desarrollo de habilidades comunicativas, intentan dar respuesta a un problema académico. En este sentido se pretende, por medio de la observación directa, la aplicación de entrevistas y el modelamiento de las relaciones sociales por medio del software UCINET, obtener información desde una mirada holística de las formas de relación en un grupo colaborante, identificando sus transformaciones. Otros estudios que se han apoyado en este tipo de análisis y que orientan esta investigación los han desarrollado Matzat, (2001), Molina, José (2001) y Wasserman (1994).

\section{Definición operacional del desarrollo conceptual}

Se entiende por desarrollo conceptual la transformación de la estructura cognitiva a partir de la asimilación obliterativa, subordinada, supraordenada o combinatoria de nuevas ideas (Ausubel, Novak y Hanesian, 2006/1983). Existen diferentes instrumentos que nos permiten obtener información de la transformación de esta estructura cognitiva. La más reconocida, en los ámbitos pedagógico y psicológico, es la representación de ésta en mapas conceptuales. Sin embargo, como afirma Vigotsky (1978), la construcción de conocimiento no es solo un proceso cognitivo sino que se produce en la interacción de los sujetos donde el lenguaje tiene un papel fundamental, tanto en la trasformación del sentido colectivo como en la referencia a la estructura conceptual de cada sujeto, por esta razón es posible extraer del discurso, ya sea hablado o escrito, una imagen del desarrollo conceptual a partir del análisis del discurso.

\section{Momentos metodológicos}

1. La primera parte del proceso se dedica a la determinación de las unidades de aprendizaje, sus objetivos, materiales de lectura y laboratorios, actividades grupales e individuales, criterios y procedimientos de evaluación en el área de tecnología y, por otro lado, a la identificación del estado inicial de las variables, con instrumentos de corte cualitativo.

2. Diseño del sitio web Portal de Visibilidad en concordancia con el diseño curricular, de modo que permita develar las producciones de los trabajos individuales y de grupo que se consideren fundamentales en el proceso de desarrollo curricular. Este dispositivo cuenta con un perfil de cada miembro y de los grupos que se organicen en la medida en que se consoliden. El sitio permite recibir comentarios y sugerencias de los visitantes y llevar una estadística de éstos. Está abierto a los estudiantes del curso, profesores, directivos y padres de familia.

3. Registro de la actividad curricular con los dos cursos, con la diferencia de que el grupo control no tiene el sitio web de visibilización.

4. Para evaluar las transformaciones de la variable de trabajo colaborativo en la comunidad de aprendizaje se aplica la metodología de Análisis de Redes Sociales, (ARS) y, adicional a 
esto, un análisis de instrumentos aplicados para obtener información referente a la red de los participantes. Con base en la elaboración de trabajos, la participación y la opinión de los miembros se elabora la matriz de interacción de la comunidad, y a partir de ésta, la representación gráfica de las relaciones sociales.

5. Toma de registro etnográfico en los diferentes momentos de la recolección de información, utilizando diarios de campo, entrevistas etnográficas y encuestas.

7. Análisis de los resultados cuantitativos en las pruebas de conocimiento, para lo cual se utilizarán métodos estadísticos como la prueba T de student y análisis de varianza.

8. Análisis cualitativo a partir de la emergencia de categorías utilizando la información de los instrumentos cualitativos (entrevista etnográfica, diarios de campo, encuestas) y la modelación a partir del software Atlas-ti, haciendo énfasis en el método análisis del discurso.

9. Triangulación de información entre los resultados de los análisis cuantitativo y cualitativo.

10. Interpretación y explicación del fenómeno por medio de la corroboración de las hipótesis planteadas.

Variable independiente: la visibilidad.

Variables dependientes: el desarrollo conceptual, el trabajo colaborativo.

\section{RESULTADOS PARCIALES}

Esta ponencia se centra en exponer los alcances del proyecto en términos de la dinámica de la comunidad de aprendizaje desde un ARS.

Para el desarrollo del proyecto se hace necesario generar un dispositivo que permita hacer visibles los productos académicos de los estudiantes pertenecientes al grupo experimental del entorno académico seleccionado. Para tal fin, se determina trabajar en un entorno de red de carácter social que posibilite la intercomunicación de usuarios, además de identificar el rol de "moderador" de los temas que puedan surgir dentro de los procesos de enseñanza y aprendizaje y, en fin, potencializar, desde el aspecto técnico, la opción de generar, compartir, debatir y verificar la información que se considere pertinente alrededor de la temática propuesta desde el aula de clase con miras a tener referentes claros sobre cuales desarrollar la medición pertinente acerca del trabajo colaborativo surgido dentro del grupo citado y, desde su participación asertiva, poder establecer criterios de avance en el desarrollo conceptual.

A partir de los datos obtenidos en el Portal de Visibilidad se presentan los siguientes resultados: 
En un primer momento los datos muestran algunos comportamientos que permiten explicar desde un ARS el comportamiento del grupo (red) y la relación de sus estudiantes (nodos). A continuación se muestra un análisis hecho al grupo experimental y al grupo control.

El procedimiento se basó en la construcción de las matrices de relaciones del grupo control y el grupo experimental (ver tabla 1 y 2), estas matrices se consolidaron con las relaciones que presentaron los estudiantes en una actividad referente a la temática "calentador solar". Esta información se recolectó por medio de un test que permitió obtener datos referentes a la relación académica entre los estudiantes en la actividad propuesta.

Tabla 1. Matriz de relación (Grupo control)

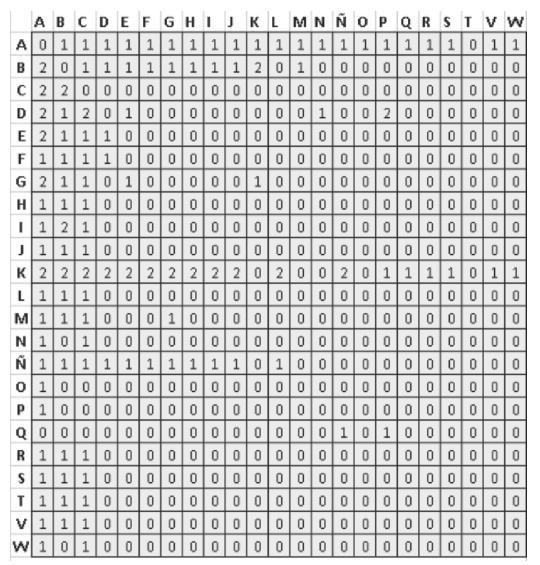

Tabla 2. Matriz de relación (Grupo Experimental)

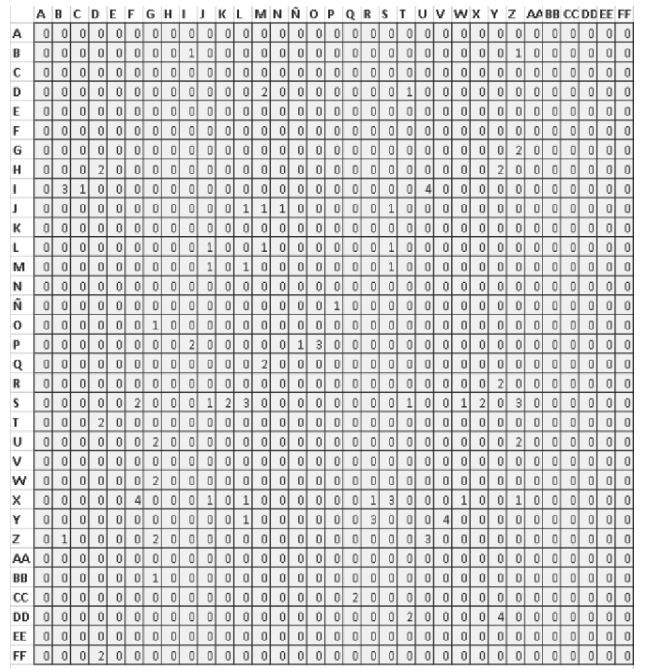


Ya consolidadas la matrices en donde se plasmó la relación académica se procedió a realizar los grafos o sociogramas que ilustran de manera gráfica las relaciones entre los nodos (estudiantes). En la figura 1 y 2 se ve la representación hecha en el programa Ucinet. Esta representación muestra los grafos de las relaciones académicas en los estudiantes de los dos grupos de trabajo, los puntos representan a los estudiantes y las líneas las relaciones entre ellos en una primera toma de datos.

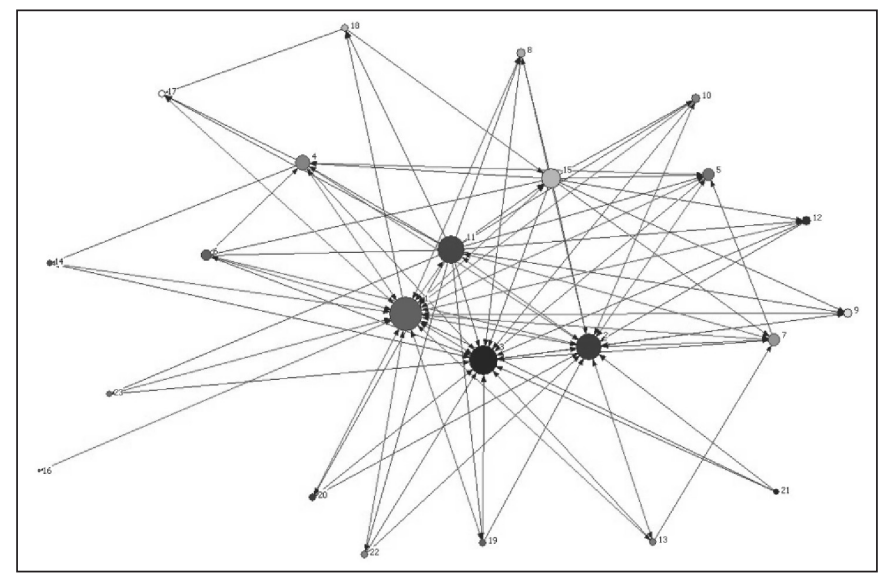

Figura 1. Grafo grupo experimental

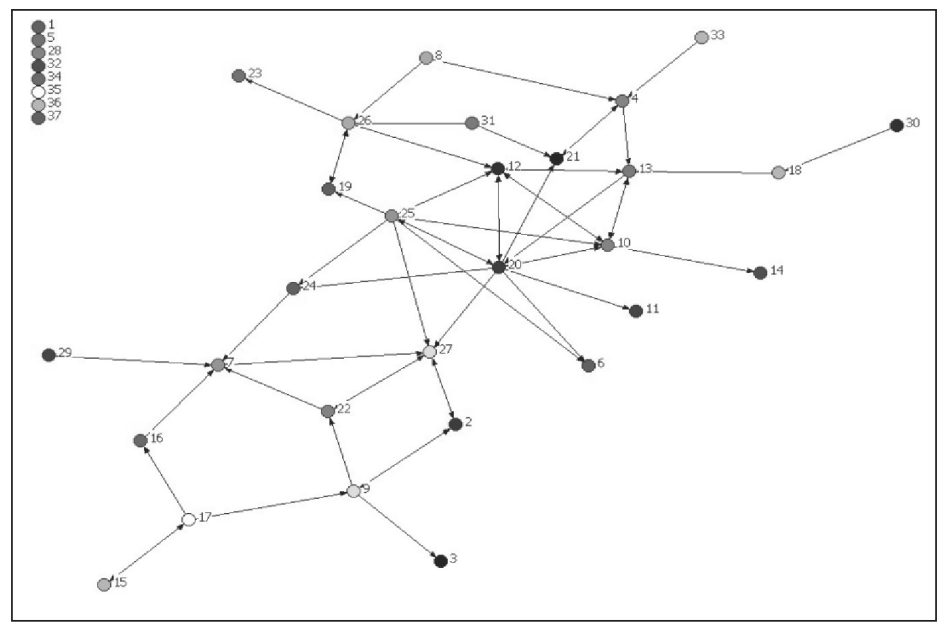

Figura 2. Grafo grupo control

En los grafos se pueden observar diferencias bien marcadas en términos de relaciones entre los estudiantes. El grupo que se somete a la estrategia de visibilidad manifiesta mayor número de relaciones entre sus participantes y un nivel de liderazgo entre los mismos, a diferencia del grupo que no desarrolla estrategia de visibilidad que presenta menor número 
de relaciones, más nodos aislados y pocas interacciones. Estas son algunas predicciones dentro del comportamiento de los grupos modelados, es necesario hacer un trabajo mucho más riguroso para poder extraer otro tipo de conclusiones frente a la dinámica de cada red. Dentro de los ARS que se utilizaron en esta parte del trabajo, se hizo uso de las medidas de centralidad, como el Degree para establecer el número de personas con las que interactúa cada participante. Con esta medida se establece el liderazgo que una persona tiene dentro de la red, ya que las diferencias entre los dos grupos están dadas en términos de la cantidad de conexiones dentro de la red.

\section{PERSPECTIVAS DEL TRABAJO}

La estrategia de visibilidad concebida como estrategia de trabajo pedagógico, puede llegar a responder a las exigencias de nuevos medios y sistemas de trabajo acordes con la tesis de Von Krogh, G. and Roos (1999), quienes sostienen la tesis que, en la era de la información, los cambios sociales, culturales y tecnológicos presentan nuevos retos a la manera de conocer y comprender. Específicamente, obligan a ir más allá del interés por los actores individuales y a poner en consideración una epistemología con dimensiones sociales de trabajo colaborativo.

La estrategia de visibilidad ha resultado útil para promover el desarrollo psicológico y cognitivo de los participantes en el contexto del concepto de Zona de Desarrollo Próximo (ZDP) tal como lo expone Vigotsky, (1978) ya que potencia la interacción para la resolución de problemas de forma colectiva.

\section{DISCUSIÓN}

El abordaje del tema de la visibilidad académica que se realizó en la presente investigación, tanto a partir del Software que se diseñó específicamente para ello, como del mensaje que implica en cuanto a desarrollar actitudes favorables e intereses de valía personal, demostró utilidad y resultó acorde con los planteamientos teóricos que abogan por una educación interactiva, colaborativa de tipo comunitario, más que individual. (Nonaka y Konno, 1998; Dillenbourg, 1999 y Hoppe y Ploetzner (1999).

El terreno de las TIC permite aprovechar y canalizar los recursos de Hadware y Software que tiene la mayoría de instituciones educativas y que comúnmente quedan subutilizados o como recursos de apoyo didáctico; la estrategia utilizada corresponde a las exigencias formativas y comunicativas de una sociedad informatizada.

El sistema de trabajo propuesto responde a los fundamentos generales del constructivismo aplicado a la educación y al enfoque socio culturalista de Vigotsky. El ambiente computacional permite que en la medida en que se hacen visibles las producciones académicas de los estudiantes, estas estén en condiciones de ser valoradas por todos los actores del proceso educativo (estudiantes, docentes, directivos, padres de familia, entre otros). 
Bajo este modelo, el maestro es un facilitador y complejizador que contribuye al desarrollo de capacidades de los estudiantes para pensar, idear, crear y reflexionar. Los actores educativos se asumen como personas capaces de tomar decisiones y emitir juicios de valor, lo que implica la participación efectiva de profesores y estudiantes que interactúan en el desarrollo de la clase para construir, crear, facilitar, liberar, preguntar, criticar y reflexionar sobre la comprensión de las estructuras profundas del conocimiento. El intercambio de ideas, los análisis y discusiones que se dan al interior de un grupo de trabajo, enriquecen en mayor grado y en menor lapso de tiempo, que cuando se intenta llegar a soluciones por sí mismo, como bien lo presenta Moll, (1990) refiriéndose al planteamiento de Vigotsky, "la ejecución que hace un niño sin ayuda no aprovechará las importantes diferencias en el funcionamiento mental que se pueden identificar analizado cómo responde el niño a la ayuda de adultos o de compañeros más capaces".

En este estudio se ha considerado importante hacer un seguimiento riguroso de la red social que se configura a partir de la visibilidad de la producción académica de los estudiantes. Esta comunidad de aprendizaje, como lo hemos llamado, obtiene unas configuraciones que ameritan rigurosidad en sus procesos de análisis, incorpora procesos estadísticos que permiten reunir, desde la teoría de grafos, aplicaciones matemáticas para cuantificar los relaciones sociales y poderlas cruzar con un componente cualitativo que da valor al tipo de relaciones entre los actores.

\section{CONCLUSIONES}

El tratamiento pedagógico de la visibilidad dinamiza los procesos de aprendizaje en la escuela, aporta a los procesos de aprendizaje desde el desarrollo conceptual y habilita la colaboración en los procesos de construcción conjunta de conocimiento.

Un estudiante que hace visible sus producciones académicas, habilita la interacción con sus compañeros y con otros actores del proceso educativo, generando cambios en su estructura conceptual, que se manifiestan en la cualificación de su discurso escrito y hablado.

La visibilidad de las producciones académicas genera un reconocimiento de los estudiantes que configura un estatus ante el contexto comunitario y se convierte en activador de colaboración en los procesos de aprendizaje.

La incorporación de un dispositivo de visibilidad mediado por TIC en los procesos de aprendizaje, permite dinamizar las clases y modelar el conocimiento que se produce entre los actores del proceso educativo.

Una persona que comparte con sus pares tiene mayor posibilidad de aprender, que una persona que trabaja desde la perspectiva individual. 
Hacer visibles las producciones académicas genera un reconocimiento de los individuos ante la comunidad que evidencia las fortalezas y debilidades de las personas, habilitando con mayor pertinencia la colaboración.

Un estudiante que hace visible sus producciones académicas, tiene la posibilidad de recibir comentarios de éstas, no solo de su profesor, sino también de sus propios compañeros, habilitando mayores oportunidades para fortalecer su aprendizaje.

\section{REFERENCIAS BIBLIOGRÁFICAS}

AMAR, José. (1989), "Infancia y calidad de vida." “Anuario Científico” Vol. n.o 3, Universidad del Norte, Barranquilla.

AUSUBEL-Novak-Hanesian. (1983/06), Psicología educativa: un punto de vista cognoscitivo. 2. a Ed. TRILLAS, México.

BANDURA, A. (1986), Social foundation of thought and action. Englewood Cliffs, NJ: Prentice Hall.

CAMPBELL, David. (1993). Good leaders are credible leaders Research Technology Management; Sep/Oct; 36, 5; ABI/INFORM Global.

DILLENBOURG, (1999), What do you mean by collaborative learning? University of Geneva, Switzerland. (Ed) Collaborative-learning: Cognitive and Computational Approaches. (pp.1-19). Oxford: Elsevier.

FISHERMAN, Shraga (2004), Ego identity and spiritual identity in religiously observant adolescents in Israel Religious Education. Decatur: Fall 2004.Vol.99, Iss. 4; pág. 371, 14 págs. Fall 2004.Vol.99, Iss. 4; 371, 14 págs.

HOPPE Y PLOETZNER (1999) Can Analytic Models Support Learning in Groups? In P.

DILlENBOURG (Ed) Collaborative-learning: Cognitive and Computational Approaches., pp. 147-169, Advances in Learning and Instruction series, Pergamon, Elsevier.

MATZAT, Uwe, (2001), Social networks and cooperation in electronic communities: a theoretical-empirical analysis of academic communication and Internet discussion groups, Dissertaties - Rijksuniversiteit Groningen.

MALDONADO y Col., (2008) Gestión de Conocimiento, visibilidad del desarrollo científico. Bogotá, Ediciones Hispanoamericanos.

MOLINA, José Luis. (2001), El análisis de redes sociales: una introducción. Ediciones Bellaterra. 
MOLL, Luis C. (1990), La Zona de desarrollo próximo de Vigotsky: una reconsideración de sus implicaciones para la enseñanza. Universidad de Arizona.

NONAKA and KONNO. (1998), The concept of Ba: Building a Foundation for Knowledge Creation. California Mangement Review, 40/3 (Spring 1998) .

VIGOTSKY, L. (1978), Mind in society. Cambridge, M. A.: Harvard University Press.

VON KROGH, Georg. And ROOS, Johan. (1999), Managing Knowledge: Perspectives on Cooperation and Competition by Georg von Krogh and Professor Johan Roos (Paperback - Dec. 4, 1996).

WASSERMAN, Stanley and FAUST, Katherine. (1994), Social Network Analysis, Methods and applications. Cambridge University.

ZAMORA, Marcelo. (2001), Ponencia en las Jornadas sobre Gestión en Organizaciones del Tercer Sector en la Universidad Di Tella, Buenos Aires, Argentina. 